\title{
Feedback-stabilized dynamical steady states in the Bose-Hubbard model
}

\author{
Jeremy T. Young $\odot,,^{1,2, *}$ Alexey V. Gorshkov $\odot,{ }^{3,4}$ and I. B. Spielman $\oplus^{3, \dagger}$ \\ ${ }^{1}$ JILA, NIST and Department of Physics, University of Colorado, Boulder, Colorado 80309, USA \\ ${ }^{2}$ Center for Theory of Quantum Matter, University of Colorado, Boulder, Colorado 80309, USA \\ ${ }^{3}$ Joint Quantum Institute, NIST/University of Maryland, College Park, Maryland 20742, USA \\ ${ }^{4}$ Joint Center for Quantum Information and Computer Science, NIST/University of Maryland, College Park, Maryland 20742, USA
}

(Received 23 June 2021; accepted 20 September 2021; published 27 October 2021)

\begin{abstract}
The implementation of a combination of continuous weak measurement and classical feedback provides a powerful tool for controlling the evolution of quantum systems. In this paper, we investigate the potential of this approach from three perspectives. First, we consider a double-well system in the classical large-atom-number limit, deriving the exact equations of motion in the presence of feedback. Second, we consider the same system in the limit of small atom number, revealing the effect that quantum fluctuations have on the feedback scheme. Finally, we explore the behavior of modest-sized Hubbard chains using exact numerics, demonstrating the neardeterministic preparation of number states, a tradeoff between local and nonlocal feedback for state preparation, and evidence of a feedback-driven symmetry-breaking phase transition.
\end{abstract}

DOI: 10.1103/PhysRevResearch.3.043075

Equilibrium is a concept central to many-body physics, both classical and quantum. It is not surprising then, that understanding new kinds of equilibrium and discovering breakdowns to equilibration are driving significant progress in physics. Recent examples include many-body states in driven-dissipative quantum systems [1-12] and the generalized Gibbs ensemble describing the limited equilibration possible for integrable quantum systems [13-21], while quantum glasses exhibit extremely long relaxation times [22,23] and many-body localized systems never equilibrate [24-33]. Here we focus instead on many-body quantum systems maintained in the dynamical steady state-a kind of generalized equilibrium - stabilized by the interplay of unitary dynamics, minimally destructive quantum measurement, and classical feedback [34-49].

Statistical mechanical equilibrium is based on the straightforward observation that a system's eigenstates are probabilistically occupied according to the thermal Boltzmann distribution, subject to any relevant constraints. The resulting density operator affords a time-independent description of the system as a thermal-equilibrium steady state. Conversely, any system described by a time-independent density operator may be associated with thermal equilibrium for an effective Hamiltonian proportional to the logarithm of that density operator, although detailed balance may not necessarily be satisfied. In

\footnotetext{
*jeremy.young@colorado.edu

†ian.spielman@nist.gov; http://ultracold.jqi.umd.edu
}

Published by the American Physical Society under the terms of the Creative Commons Attribution 4.0 International license. Further distribution of this work must maintain attribution to the author(s) and the published article's title, journal citation, and DOI. this sense then, is it possible to identify and explore systems maintained in low-entropy steady states associated with exotic effective Hamiltonians with highly nonlocal or $N$-body interactions? Optical pumping [50] and laser cooling [51]both described by the physics of open quantum systems - are iconic examples where large ensembles of atoms enter lowentropy steady states well described by single-atom physics with little correlation between atoms.

In contrast, quantum error correction (QEC) codes are highly specialized and sophisticated examples of measurement-feedback systems that can dynamically stabilize strongly entangled arrays of qubits [52,53]. A digital quantum computation device consists of an interconnected collection of qubits, and QEC utilizes multiqubit syndrome measurements, often realized via ancilla qubits that are first coupled to the error-prone qubits and then measured. The coupling and measurement are designed to be sensitive to select errors, but not to the quantum state involved in computation. In some forms of QEC [54], the classical information resulting from measuring the ancilla can inform subsequent error-correcting feedback stages, thereby maintaining the quantum computation device in a type of dynamical steady state.

Quantum state preparation is closely related to QEC: both attempt to drive a system toward a particular state. In the case of QEC, this is the state prior to an error; in the case of state preparation, it is a particular state of interest. State preparation is particularly important in the field of metrology $[55,56]$. By preparing highly entangled states, such as squeezed states [35,36,57-61], it becomes possible to make measurements that are far more accurate than in an unentangled classical system. While a variety of sophisticated techniques have resulted in experimental measurements with incredible accuracy, incoherent effects often provide a fundamental limitation. Like with typical QEC for quantum computation, measurement 
and feedback may provide a means of reducing the problems introduced by incoherent processes [62-72].

Here, we study this problem from three perspectives progressively increasing in complexity. (1) We begin by considering a minimal two-site system in the large-atom-number classical limit—a type of nonlinear top-and derive the exact equations of motion including feedback. This model predicts what types of feedback can drive the system into different fixed points, providing guidance for what type of quantum states will appear. (2) We then consider a stochastic Schrödinger equation description of the same double well. By investigating both the classical large-atom limit and the quantum small-atom limit, we connect the classical fixed points to their associated quantum dynamical steady states. (3) We conclude with the numerically exact time dynamics for modest-sized Hubbard chains in the quantum low-density limit (see Refs. [40] and [41] for investigations in the more classical high-density limit) and show that nearly arbitrary distributions of number states can be near-deterministically prepared and that nonlocal feedback algorithms can both improve fidelity and accelerate state preparation. Moreover, we show that the corresponding feedback can be modified in a simple way to realize a symmetry-breaking transition.

From a broader perspective, this paper shows that straightforward feedback derived from highly idealized continuous quantum measurements can stabilize low-entropy dynamical steady states. It is the task of future work to expand upon the impact of increasingly realistic experimental parameters.

\section{MODEL}

Here we focus on the idealized 1D Bose-Hubbard model subjected to continuous weak measurement $[73,74]$ to illustrate the possible dynamical steady states in many-body quantum systems. As depicted in Fig. 1, this model consists of a 1D Bose-Hubbard chain (the system) dispersedly coupled to a transverse laser field (reservoir), measured via phase contrast imaging (optical homodyne detection), and with local tunneling strengths and on-site potentials that are dynamically adjusted based upon the measurement outcome (feedback). At time $t$, the Bose-Hubbard chain is governed by the system Hamiltonian

$$
\hat{H}_{\mathcal{S}}=\sum_{j}-J_{j}\left(\hat{a}_{j+1}^{\dagger} \hat{a}_{j}+\text { H.c. }\right)+V_{j} \hat{n}_{j}+\frac{U}{2} \hat{n}_{j}\left(\hat{n}_{j}-1\right)
$$

expressed in terms of the bosonic field operators $\hat{a}_{j}^{\dagger}$ describing the creation of an atom at site $j$ and $\hat{n}_{j}=a_{j}^{\dagger} a_{j}$ the number operator at site $j$, with positive real-valued tunneling strength $J_{j}(t)$ between sites $j$ and $j+1$, on-site energy $V_{j}(t)$, and interaction strength $U$. The chemical potential is absent in this microcanonical ensemble study.

\section{A. Measurement model}

The system is coupled to the reservoir by the systemreservoir Hamiltonian

$$
\hat{H}_{\mathrm{SR}}=\hbar g \sum_{j} \hat{a}_{j}^{\dagger} \hat{a}_{j} \otimes \hat{b}_{j}^{\dagger} \hat{b}_{j}
$$

that describes a dispersive off-resonance interaction of light with two-level atoms detected by homodyne measurement
System

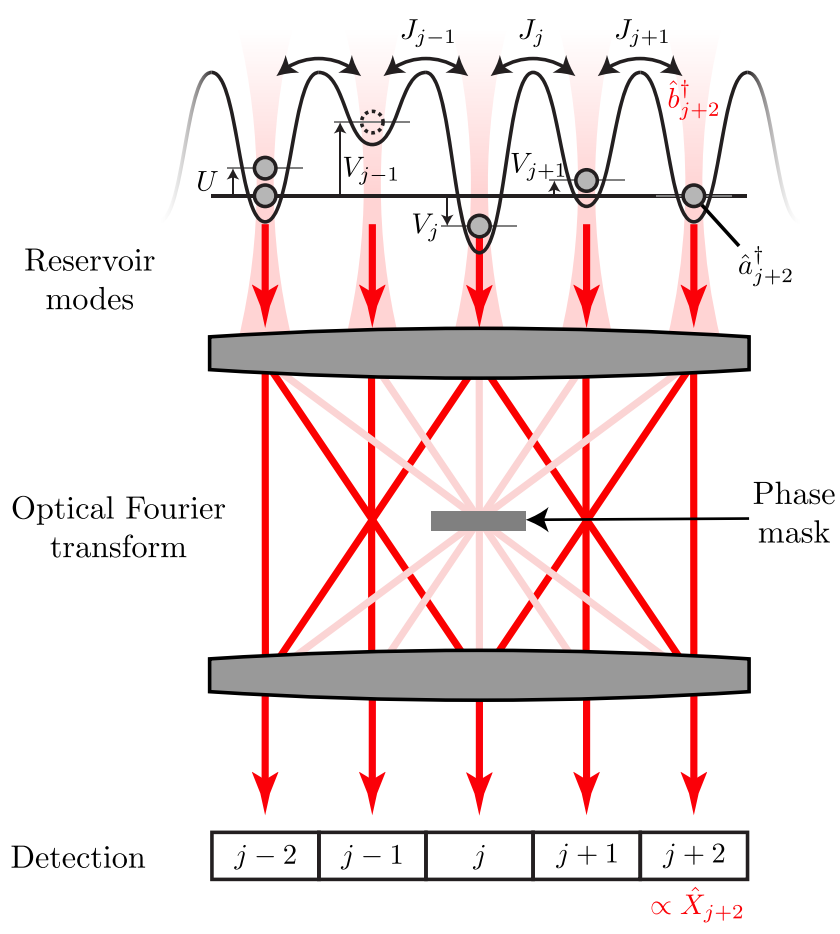

FIG. 1. System and measurement schematic. An ensemble of locally interacting (with strength $U$ ) neutral atoms described by the bosonic field operators $\hat{a}_{j}$ are confined in a lattice potential with tunneling strength $J_{j}$ between sites $j$ and $j+1$. These atoms interact with independent modes of an optical field described by bosonic field operators $\hat{b}_{j}$. After interacting, the optical modes are imaged via phase contrast imaging, implementing a spatially resolved optical homodyne detection providing a measurement sensitive to the quadrature field operator $\hat{X}_{j}=\left(\hat{b}_{j}+\hat{b}_{j}^{\dagger}\right) / 2$. This idealized measurement assumes that the incident laser field is only forward scattered, essentially making the paraxial approximation.

[75] or, equivalently, phase contrast imaging, shown in Fig. 1. Here, the bosonic field operator $\hat{b}_{j}^{\dagger}$ describes the addition of a photon into mode $j$ associated with lattice site $j$ (here we focus on an idealized case with mode functions associated one-to-one with lattice sites), $g$ captures the strength of the system-reservoir coupling, and the reservoir modes $\hat{b}_{j}$ are each in the coherent state $|\alpha\rangle$ prior to interacting with the system. Physically, this operator describes rotations in the $\hat{X}_{j}-\hat{P}_{j}$ quadrature plane for each reservoir mode, in proportion to the local number of atoms. After interacting with the system for a time $t_{m}$, the reservoir modes are projectively measured by optical homodyne measurement (in practice implemented by phase contrast imaging), giving access to the $\hat{X}_{j}=\left(\hat{b}_{j}^{\dagger}+\hat{b}_{j}\right) / 2$ quadrature observables.

A strong measurement on the reservoir in effect affords a single weak measurement of the system observables $\hat{n}_{j}$ with strength governed by $g$, the reservoir field amplitude $\alpha$, and the measurement time $t_{m}$. A measurement outcome

$$
n_{j}(t)=\left\langle\hat{n}_{j}(t)\right\rangle+\delta n_{j}
$$


at time $t$ contains a contribution from the system observable's instantaneous expectation value $\left\langle\hat{n}_{j}(t)\right\rangle$, along with a noise contribution $\delta n_{j}=m_{j} / \varphi$ from projectively measuring the reservoir coherent state $\left|\alpha_{j}\right\rangle$. Here $m_{j}$ is a classical random variable with zero mean $\overline{m_{j}}=0$ and variance $\overline{m_{j} m_{j^{\prime}}}=\delta_{j, j^{\prime}} / 2$. We consider an optical mode function consisting of a traveling pulse of light with extent $c t_{m}$, where $c$ is the speed of light. Converting from the continuum mode functions to this compact function introduces a factor of $1 /\left(c t_{m}\right)$ into the definition of the generalized measurement strength parameter. This leads to the generalized measurement strength parameter $\varphi^{2}=g^{2}|\alpha|^{2} t_{m} / c$, defining the measurement noise $\overline{\delta n_{j}^{2}}=$ $\Delta n^{2}=1 / 2 \varphi^{2}$ and the backaction of this measurement on the system as described by the Kraus operator [76],

$$
\hat{K}\left(n_{j}\right)=\exp \left[-\sum_{j} \frac{\varphi^{2}\left(\hat{n}_{j}-n_{j}\right)^{2}}{2}\right],
$$

conditioned on the measurement outcomes $n_{j}$. Given an initial state $|\psi\rangle$ and a measurement outcome $n_{j}$, the postmeasurement state of the system is given by $\hat{K}\left(n_{j}\right)|\psi\rangle$. As one might intuitively expect from a simple classical measurement, this gives a distribution of measurement outcomes with a width $\propto 1 / \varphi$ that decreases linearly with the system-reservoir coupling $g$ but as the square root of the measurement time $t_{m}$ and intensity of the probe field $|\alpha|^{2}$. In the following analysis, we consider the continuous limit of many such weak measurements, taking the time between subsequent measurements to be $t_{m}$ and applying measurements with a strength associated with a measurement time $t_{m}$.

\section{B. Feedback model}

For sufficiently weak measurements, the quantum projection noise $\delta n_{j}$ present in any individual measurement can greatly exceed the contribution of the instantaneous expectation value $\left\langle\hat{n}_{j}(t)\right\rangle$. As a result, we borrow from classical control theory [77] and derive an error signal $\epsilon(t)$ from a temporal low-pass filter,

$$
\tau \dot{\epsilon}_{j}(t)+\epsilon_{j}(t)=n_{j}(t),
$$

applied to the direct measurement outcomes. This consists of an integrator with a low-frequency gain limit, with time constant $\tau$. This filter retains the low-frequency $\lesssim 1 / \tau$ components of $n_{j}(t)$ (containing contributions from both system dynamics and projection noise), but rejects the high-frequency components (dominated by projection noise). Here we assume that the measurement time $t_{m} \ll \tau$, implicitly making Eq. (3) a stochastic differential equation, see Ref. [41] for a more detailed discussion. The resulting error signal $\epsilon_{j}(t)$ thus approximates the continuous measurement limit of a sequence of weak measurements of $n_{j}(t)$ with measurement times $\tau$ regardless of the physical measurement time $t_{m} \ll \tau$. Note that because this filter is applied in real time, $\epsilon_{j}(t)$ will lag behind $n_{j}(t)$ by $\tau$, and we take $\epsilon_{j}(0)=0$ in our simulations. Because the goal is neither to perform quantum state estimation nor to drive the system into a predefined state, we do not employ Kalman filter techniques [78].

Here we focus on possible dynamical steady states when this classical information is then fed back into the Hamiltonian in one of two ways. We shift either the on-site energy

$$
V_{j}(t)=V_{0}+\delta V_{j}(t)=V_{0}+G_{V} \epsilon_{j}(t)
$$

in proportion to the local feedback signal (with gain $G_{V}$ ), or the tunneling

$$
J_{j}(t)=J_{0}+\delta J_{j}(t)=J_{0}+G_{J}\left[\epsilon_{j}(t)-\epsilon_{j+1}(t)\right]^{2}
$$

based on the difference along that link (with gain $G_{J}$ ). These two forms of feedback are the most simple physically realistic options: the potential $V_{j}(t)$ simply shifts in proportion to the error signal; however, because negative tunneling is difficult to achieve, quadratic feedback is the most simple realistic feedback to the tunneling term. Both of these can be realized using quantum gas microscopes $[79,80]$ where a digital mirror device can locally control both the intensity of the lattice light (increasing or decreasing tunneling [81]) as well as changing the intensity on the lattice sites (altering the on-site potential [30]).

\section{TWO-SITE LATTICE}

The two-site Bose-Hubbard model with $N$ atoms can be recast as an $f=N / 2$ angular momentum system with mappings $\hat{n}_{1}-\hat{n}_{0} \rightarrow 2 \hat{F}_{z}, \hat{a}_{1}^{\dagger} \hat{a}_{0}+\hat{a}_{0}^{\dagger} \hat{a}_{1} \rightarrow 2 \hat{F}_{x}$, and $\hat{a}_{1}^{\dagger} \hat{a}_{0}-$ $\hat{a}_{0}^{\dagger} \hat{a}_{1} \rightarrow 2 i \hat{F}_{y}$, that obey a dimensionless angular momentum commutation relation $\left[\hat{F}_{i}, \hat{F}_{j}\right]=i \epsilon_{i j k} \hat{F}_{k}$. This gives the Hamiltonian

$$
\hat{H}_{\mathcal{S}}=-2 J \hat{F}_{x}+\Delta \hat{F}_{z}+U \hat{F}_{z}^{2},
$$

where we have absorbed constant terms into an overall shift in the zero of energy and defined $\Delta=V_{1}-V_{0}$. This also results in the single filter equation

$$
\tau \dot{\epsilon}(t)+\epsilon(t)=2 f_{z}(t)
$$

for $\epsilon(t) \equiv \epsilon_{1}(t)-\epsilon_{0}(t)$ and feedback equations

$$
\Delta(t)=\Delta_{0}+G_{V} \epsilon(t) \text { and } J(t)=J_{0}+G_{J} \epsilon(t)^{2},
$$

which are slightly adjusted with an additional factor of 2 on the right-hand side of the filter equation, resulting from the details of the angular momentum mapping.

The on-site measurement outcomes at time $t$ can be reexpressed as

$$
f_{z}(t)=\left\langle\hat{F}_{z}(t)\right\rangle+m /(\sqrt{2} \varphi),
$$

with contribution [82] from the instantaneous expectation value $\left\langle\hat{F}_{z}(t)\right\rangle$. As before, the noise is defined by a classical random variable $m$ with zero average $\bar{m}=0$ and variance $\overline{m^{2}}=1 / 2$. Backaction is described by the Kraus operator:

$$
\hat{K}\left(f_{z}\right)=\exp \left[-\varphi^{2}\left(\hat{F}_{z}-f_{z}\right)^{2}\right] .
$$

Taken together, these expressions provide an equivalent formulation of the two-site system in the language of angular momentum.

\section{A. Classical limit}

The Heisenberg equations of motion in the large- $N$ limit (i.e., ignoring quantum fluctuations and thus measurement 
uncertainty) reduce to the classical equations of motion,

$$
\dot{\mathbf{F}}=\left(\begin{array}{ccc}
0 & -\left(\Delta+2 U F_{z}\right) & 0 \\
\left(\Delta+2 U F_{z}\right) & 0 & 2 J \\
0 & -2 J & 0
\end{array}\right) \cdot \mathbf{F},
$$

where $\mathbf{F} \approx\left(\left\langle\hat{F}_{x}\right\rangle,\left\langle\hat{F}_{y}\right\rangle,\left\langle\hat{F}_{z}\right\rangle\right)$ denotes the classical angular momentum vector, ignoring quantum fluctuations. Note that because the interaction $U$ enters via a quartic term in the Hamiltonian, it enters the classical equations of motion nonlinearly. Thus, to properly compare systems with different $f$, we keep $U f$ fixed to a constant value. Similarly, since the potential and tunneling feedback terms behave like effective fourth- and sixth-order terms in the Hamiltonian, respectively, we must also keep $f G_{V}$ and $f^{2} G_{J}$ fixed to constant values.

Tunneling causes the macroscopic spin vector to precess around $\mathbf{e}_{x}$, a potential imbalance leads to precession around $\mathbf{e}_{z}$, and the nonlinear interaction term effectively drives precession around $\mathbf{e}_{z}$ with angular frequency in proportion to $F_{z}$. Figure 2(a) plots the energy [from Eq. (6)] and trajectories [from Eq. (11)] for $\Delta=0$ as a function of the polar angles $\phi$ and $\theta$ with respect to an $\mathbf{e}_{x}$ aligned spherical coordinate system ( $\phi=0$ in the $\mathbf{e}_{y}$ direction). The displayed trajectories illustrate the two classes of orbits: (1) Near $F_{x}=f$ (i.e., $\theta=0)$, the phase $\phi$ exhibits running solutions [83,84] orbiting around $\mathbf{e}_{x}$ : Josephson oscillations (JOs). (2) For larger $\theta$, closed orbits around $F_{x}=-J / U, F_{y}=0$, and $F_{z}^{2}=f^{2}-F_{x}^{2}$ correspond to oscillations about a density imbalanced excited state [85]: macroscopic self-trapping (MST), present only for $|J / U|<f$. For $|J / U|>f$, the two MST solutions merge to form a second stable $\mathrm{JO}$ fixed point at $F_{x}=-f$.

\section{B. Linearized dynamics}

The impact of feedback is most clearly understood by first linearizing the dynamics around each of the stable fixed points of the JO and MST orbits, in general, giving elliptical orbits. The displacements $\delta F_{x, y, z}$ each obey second-order differential equations such as

$$
\ddot{\delta} F_{z}+\omega^{2} \delta F_{z}=0
$$

describing motion in an effective harmonic potential, with angular frequencies

$$
\omega_{\mathrm{JO}}^{2}=4 J_{0}\left(J_{0} \pm U f\right) \quad \text { and } \quad \omega_{\mathrm{MST}}^{2}=4\left(U^{2} f^{2}-J^{2}\right)
$$

for the JO and MST points, respectively. For $\tau=0$, the feedback described by Eq. (8) becomes proportional to $F_{z}$ and shifts the location of the fixed points (potentially even eliminating them entirely), as well as altering the frequency and ellipticity of the orbits. Still, the form of the differential equations is unchanged: no damping. In the above example, conventional damping would arise from an additional friction term $\delta \dot{F}_{z} / \tau$ and, more generally, damping (or antidamping) will occur only when odd and even derivatives are mixed. In our case, $\epsilon(t)$ mixes these derivatives when $\tau \neq 0$, changing the relationship between $\epsilon(t)$ and $f_{z}(t)$ according to Eq. (7), thus changing the effect of $\delta F_{z}$ on Eqs. (8) and (11). The effect of feedback can be quantified in linear response about both the JO and the MST fixed points, allowing us to derive a damping rate $\Gamma$ in each case, in the limit of weak damping $\omega \Gamma \ll 1$, where $\omega$ is the angular frequency of the relevant fixed point. (a) Classical trajectories

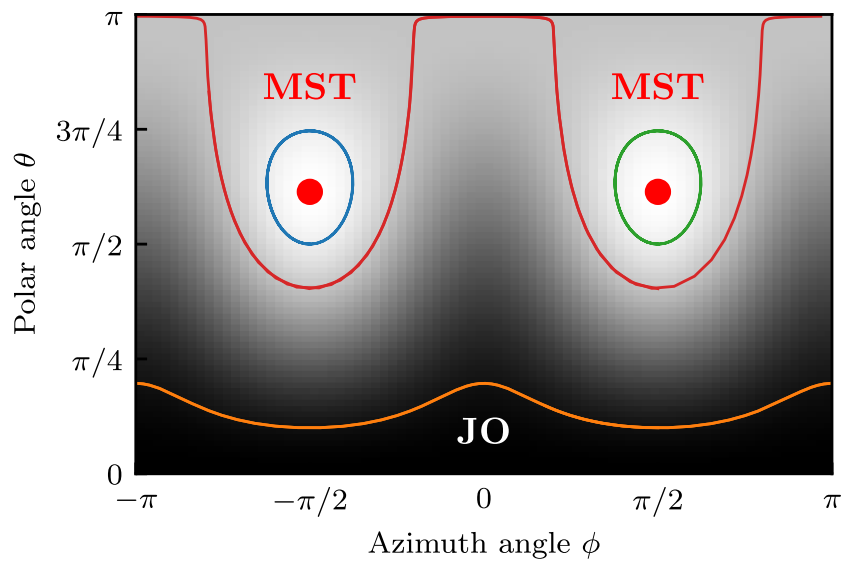

(b) Dynamics near fixed points

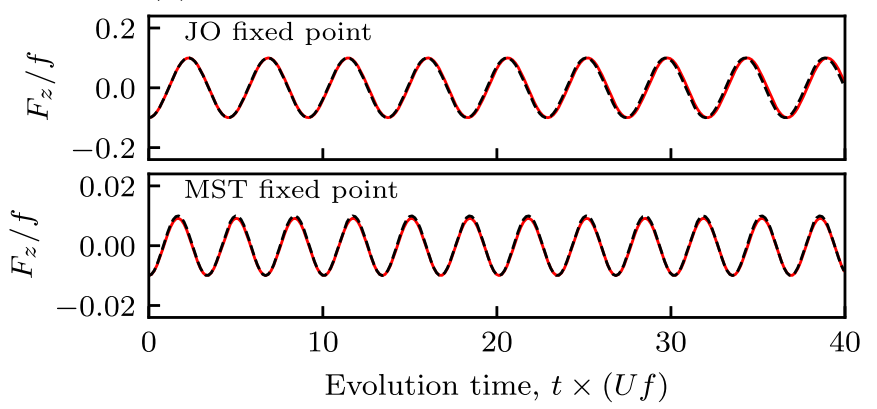

(c) Classical dynamics with feedback
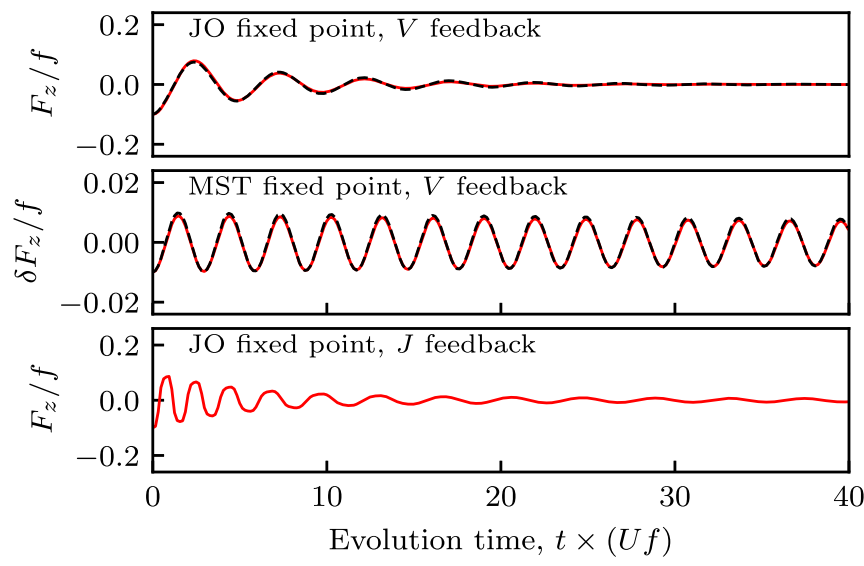

FIG. 2. Classical spin model computed for $f=32, U f=2$, $J_{0} /(U f)=0.35$, and $\Delta=0$. (a) Trajectories as a function of $\theta, \phi$ with respect to the $\mathbf{e}_{x}$ axis. These orbits are separated by the red curve into regions of Josephson oscillations (e.g., orange curve) and oscillations around the macroscopically self trapping point (e.g., blue and green curves). The color indicates energy, with black denoting the lowest energy and white the largest. (b), (c) Orbits around both JO and MST fixed points. Red curves denote the result of our numerical simulation while the black dashed curves plot the result of linear response theory. Panel (b) plots the case with no measurement or feedback and panel (c) plots the case with feedback with $\tau=1 / 2$ and $t_{m}=0.01$. Feedback in $V$ [bottom two plots in (c)] damps to the JO and MST fixed points for $f G_{V}=-1$ and $1 / 4$, respectively. Feedback in $J$ [bottom plot in (c)] damps to the JO fixed point with $G_{J}=2$. Because the feedback is nonlinear, no linear response theory is displayed in the bottom plot. 
In this limit, the damping rates are $\omega \Gamma=\gamma \omega \tau /\left(1+\tau^{2} \omega^{2}\right)$, with the most effective damping when $\omega \tau=1$, and where the strength $\gamma$ governs the system dependence of the feedback. For feedback in the potential, the resulting strengths are

$$
\gamma_{\mathrm{JO}}=-2 J_{0} f G_{V} \quad \text { and } \quad \gamma_{\mathrm{MST}}=2 \frac{J_{0}^{2} G_{V}}{U+G_{V}},
$$

showing that, depending on the sign of $G_{V}$, the system can be damped effectively. Focusing on the case of positive interactions $U>0$ and positive base tunneling $J_{0}>0$, either only the JO fixed point is stable $\left(0<-G_{V}<U\right)$, only the MST fixed points are stable $\left(0<G_{V}<U\right)$, or both types of fixed points are stable $\left(-G_{V}>U\right)$.

In contrast, for feedback in the tunneling channel, the quadratic response $\delta J=G_{J} \epsilon(t)^{2}$ leads to very slow damping as the density-balanced JO fixed point is approached, i.e., at the JO fixed point, $\epsilon(t)=0$, and the linear contribution to $\delta J$ is zero. Thus, only the MST state has damping or antidamping in linear response, and for the physical sign of the gain coefficient $G_{J}>0$, this results in antidamping.

\section{Fluctuations}

Although the classical limit omits quantum fluctuations (by assuming a spin-coherent state), it need not omit measurement backaction. A prescription similar to that of Ref. [40] for coherent states gives a final state conditioned on the random variable $m$,

$$
F_{z \mid m}=F_{z}+\sqrt{2} f \varphi m \sin ^{2} \theta_{z},
$$

for measurements of $\hat{F}_{z}$ described by Eq. (9), where $\theta_{z}$ is defined with respect to $\mathbf{e}_{z}$. This prescription takes an initial spin coherent state, then applies the Kraus operator; because the resulting state is not necessarily a spin-coherent state, we find the coherent state with the largest overlap as the updated state. The core message of this expression is that $F_{z}$ is updated as informed by the measurement outcome, but for initial states nearly polarized along $\mathbf{e}_{z}$, the state is nearly unaltered. Physically, this is expected because we are working at fixed total angular momentum $f$ and a state already polarized along $\mathbf{e}_{z}$ cannot become more polarized.

Lastly, equating Eqs. (9) and (13) suggests an optimal measurement strength for which the true value of $F_{z}$ following the measurement [Eq. (9)] is equal to the measurement outcome [Eq. (13)], i.e., the measurement outcome accurately reflects the new state of the system. This occurs only for an optimal measurement strength $\varphi_{\mathrm{opt}}^{2}=\left(2 f \sin ^{2} \theta_{z}\right)^{-1}$ that would be selected to yield optimal performance near the desired fixed point. We also note that with Eqs. (9) and (13), this implies that for the optimal measurement strength, the measurement noise and backaction both scale like $f^{-1 / 2}$, fractionally going to zero in the $f \rightarrow \infty$ limit as one would expect.

The low-pass filter from which we derive the error signal has time-constant $\tau$, which plays the role of an effective measurement time. Assuming no delay between measurements, this implies an individual-measurement coupling $\varphi_{\mathrm{opt}, 0}^{2} \simeq$ $\left(2 f \sin ^{2} \theta_{z}\right)^{-1} \times\left(t_{m} / \tau\right)$, so the $\tau / t_{m}$ measurements which take place in the time interval $\tau$ give a single average outcome with the optimal strength $\varphi_{\text {opt }}$. (a) JO free evolution, $N=64, f=32$

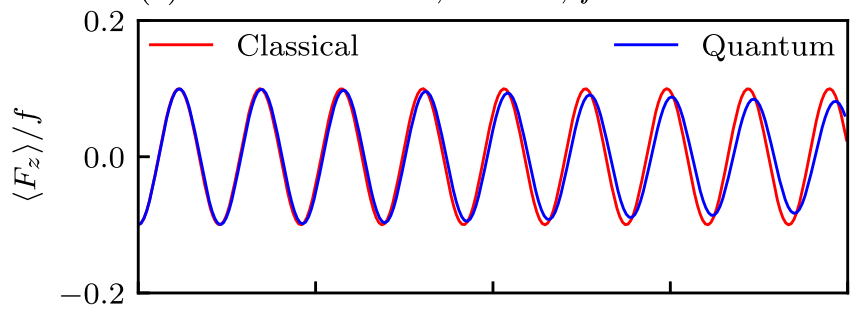

(b) JO, $V$ damping, $N=64, f=32$

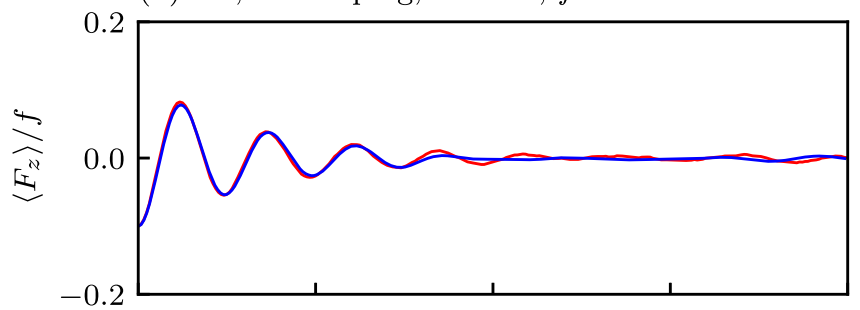

(c) JO, $V$ damping, $N=2, f=1$

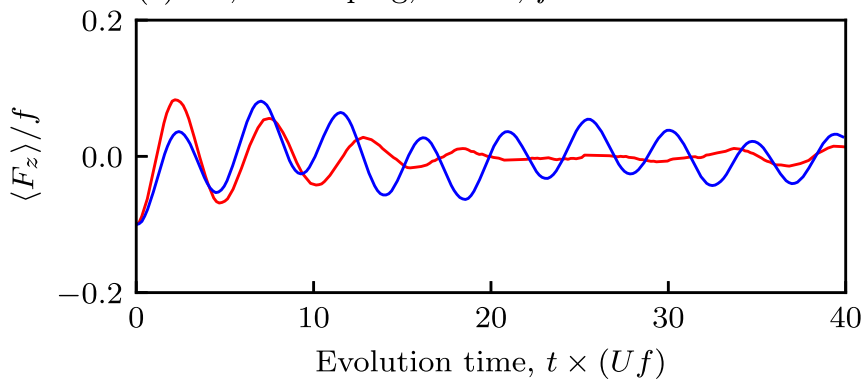

FIG. 3. Comparison of classical (red) and quantum (blue) simulations with identical parameters. (a) Free evolution around the JO fixed point for the same parameters as in Fig. 2, including $\tau=$ 0.5. (b) Evolution including measurement noise, backaction, and feedback for the same parameters as in Fig. 2, as well as a measurement strength $f^{1 / 2} \varphi=t_{m}^{1 / 2}$ with $t_{m}=0.01$. These trajectories were averaged over 1024 realizations to show their average behavior. (c) Evolution for $N=2$, with parameters suitably scaled by $f$ to yield same classical dynamics as (b).

\section{Quantum double well}

With these basic insights from the classical model in mind, we compare to a quantum-trajectories stochastic Schrödinger equation simulation of this double-well problem, including all effects of backaction resulting from measurement to the classical spin model, including measurement noise and backaction. Figure 3(a) shows the coherent evolution of a spin coherent state with $N=64$ or $f=32$, according to the classical spin model (red) and the quantum simulation (blue), with nearly perfect overlap. The agreement continues to improve with increasing $N$, as neglecting quantum fluctuations becomes an increasingly good approximation. This overlap persists in Fig. 3(b), which includes the effects of measurement, backaction, and feedback, again showing good correspondence between the classical and quantum models. Lastly, as the number $N$ is reduced from 64 to 2 (and $U$ is correspondingly increased by a factor of $64 / 2$ to keep $U f=2$ ), neglecting fluctuations becomes a very poor approximation, and the classical and quantum trajectories deviate.

The classical-limit modeling provided valuable insight in guiding parameter selection even for quantum systems: (1) 
feedback can change the dynamical steady state by introducing effective potentials and (2) the optimal filter time $\tau$ is inversely proportional to the dynamical timescale of excitations in the measurement channel. A basic intuition for the latter point comes from a continuously monitored classical harmonic oscillator. The measured position converts into knowledge of momentum one-quarter period later, allowing a time-delayed feedback force to reduce that known velocity. In the present case, $\tau$ in the low-pass filter gives the same outcome by introducing a phase shift for signals with angular frequency $\gtrsim 1 / \tau$. We certainly expect that more complex filters, proportional-integral-differential or Kalman [78] filters, for example, would give improved performance.

\section{EXTENDED LATTICE}

In this section, we extend our analysis to the case of small one-dimensional Bose-Hubbard chains. Unlike the case of two sites, there is no angular momentum map and the appropriate mean-field description is a discrete Gross-Pitaevskii equation, similar to that discussed in Refs. [40,41]. Here, we focus on the quantum region by considering states with mean densities of just a few particles per site and studying the system's behavior numerically using a quantum trajectories approach [86-88]. Correspondingly, the fluctuations in all figures are a result of sampling error. We will also consider a range of target density distributions, in which the error signal derives from the difference between the observation and the target. Throughout, we will express everything in units where the interaction strength $U=1$. Recent closely related work investigates preparing similar Mott-like states via global measurements rather than the local measurements considered here [39].

\section{A. Feedback models}

We consider two possible tunneling feedback schemes. The first is the nearest-neighbor approach introduced in Eq. (5). The second, the imbalance approach, uses highly nonlocal information, and relies instead on imbalances between the left and right sides of the system. For the double-well system considered earlier, these two schemes are identical. The tunneling strengths are, respectively,

$$
\begin{gathered}
J_{j}(t)=G\left|\left[\epsilon_{j}(t)-\epsilon_{j+1}(t)\right]-\left[N_{j}-N_{j+1}\right]\right|^{2}, \\
J_{j}(t)=G\left|\left[\epsilon_{j, L}(t)-\epsilon_{j, R}(t)\right]-\left[N_{j, L}-N_{j, R}\right]\right|^{2},
\end{gathered}
$$

where

$$
\begin{aligned}
N_{j, L} & =\sum_{k \leqslant j} N_{k}, \quad N_{j, R}=\sum_{k>j} N_{k}, \\
\epsilon_{j, L} & =\sum_{k \leqslant j} \epsilon_{k}, \quad \epsilon_{j, R}=\sum_{k>j} \epsilon_{k},
\end{aligned}
$$

and $N_{j}$ indicates the target occupation number of site $j$. Since the tunneling strength without feedback is $J_{0}=0$ and measurement occurs in the number basis, this means that the target state $\left|N_{1} N_{2} \cdots\right\rangle$ will be an evolution-free fixed point in the absence of feedback induced by noise from the measurements. Given the structure of the imbalance approach, which entails

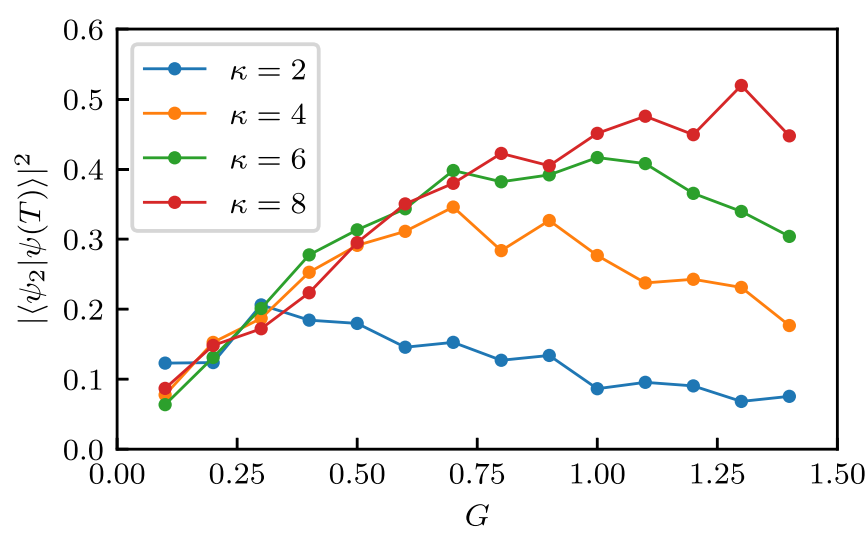

FIG. 4. Average overlap $\left|\left\langle\psi_{2} \mid \psi(T)\right\rangle\right|^{2}$ for different values of $G$ and $\kappa$ for the nearest-neighbor approach using 256 trajectories.

separating the system into left and right sides, we will consider open boundary conditions when discussing state preparation.

The purpose of the imbalance approach is to take advantage of the global knowledge of the target state, knowledge that is not used in the nearest-neighbor approach. For example, consider the scenario in which the target state is $|43210\rangle$ and the system is in the state $|04321\rangle$. In this case, the nearestneighbor approach will only initially turn on the tunneling between the first two sites and gradually turn on the other tunneling terms from left to right as the bosons move to the left. In contrast, the imbalance approach will turn on all tunneling terms and begin to transfer atoms from the right side of the system to the left. However, the use of this more global knowledge comes at a cost to the feedback uncertainty. Since $\epsilon_{j, L / R}$ involves a sum of $N$ different measurement records, the resulting uncertainty will be enhanced by a factor of $\sqrt{N}$. As a result, there is a trade-off between using global knowledge and the uncertainty in the feedback. Finally, we will discuss these two feedback approaches in terms of a continuous measurement rate $\kappa$, where $\varphi \equiv \sqrt{2 \kappa t_{m}}$ and we use $t_{m}=0.01$ in our numerical simulations.

\section{B. State preparation}

We compare the performance of these two approaches for three different target states: $\psi_{1}=|11111\rangle, \psi_{2}=|20202\rangle$, and $\psi_{3}=|30003\rangle$. We fix the filter time $\tau=0.3$ and the evolution time $T=10+\tau$ [89] in units of $U^{-1}$ and examine the behavior of the final target state overlap $\left|\left\langle\psi_{i} \mid \psi(T)\right\rangle\right|^{2}$ as a function of $\kappa$ and $G$. For each trajectory, we initialize the system in a Haar random state. While we could consider the steady-state behavior of this system, from the perspective of creating a target state in a physical system, it is more useful to consider the finite-time behavior.

Figure 4 illustrates the target state overlap for $\psi_{2}$ at different values of $\kappa$ and $G$ using the nearest-neighbor approach. The behavior for other target states and the imbalance approach are qualitatively similar. For all values of the measurement strength $\kappa$, we see that there is an optimal choice of the gain $G$ for which the overlap is maximized. This optimal value of the gain represents a balance between the need to direct the system to the desired target state quickly and the fact that measurement uncertainty can lead to errors in the 
(a)
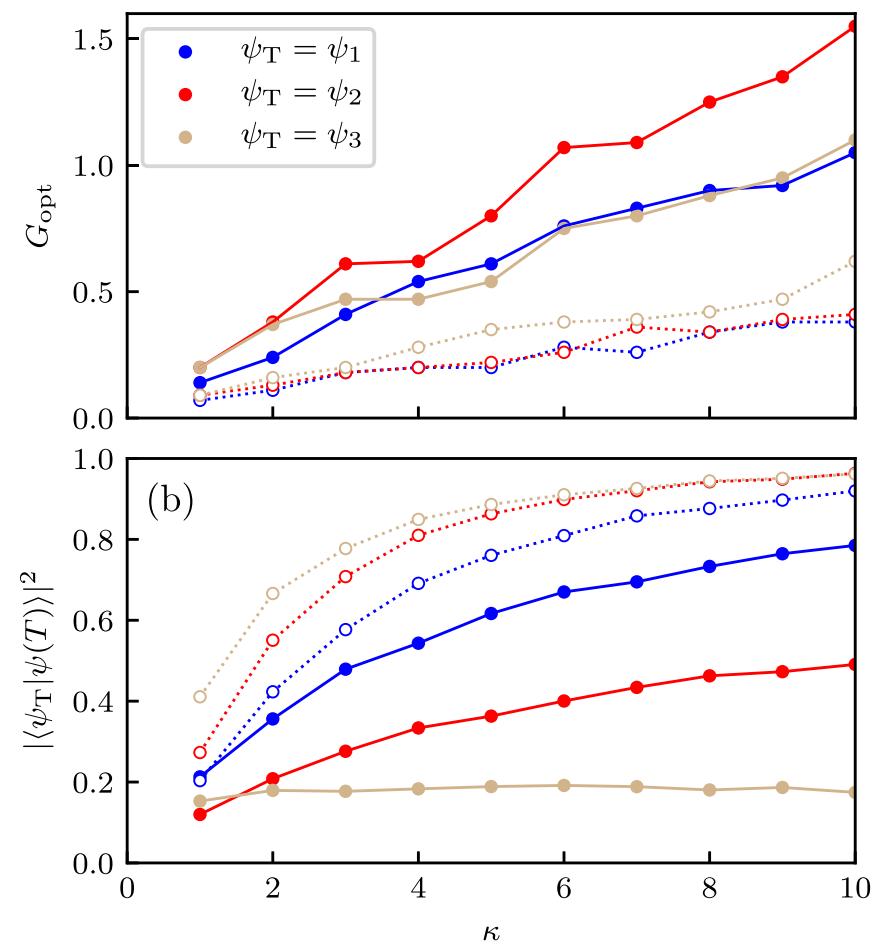

FIG. 5. Optimal behavior of the two feedback approaches using 2048 trajectories for each data point except for the nearest-neighbor approach for $\psi_{3}$, which uses 4096 trajectories. (a) Optimal gain $G_{\text {opt }}$ as a function of $\kappa$. (b) Optimal target state overlap as a function of $\kappa$. The solid (dotted) lines correspond to the nearest-neighbor (imbalance) approach. Blue, red, and tan lines correspond to $\psi_{T}=$ $\psi_{1}, \psi_{2}, \psi_{3}$, respectively.

application of the feedback. We identify the optimal value of the gain and the corresponding target state overlap for the different target states and measurement strengths in Fig. 5. Several trends can be identified in the optimal feedback behavior.

The first trend is that the optimal gain grows linearly with the measurement strength. This reflects the fact that stronger measurements correspond to more accurate knowledge of the system's state, so the system can be more strongly driven to the target state without making errors due to the measurement uncertainty. Additionally, the gain must increase with the measurement strength to avoid quantum Zeno effects, i.e., the dynamics due to the feedback must become sufficiently fast so the repeated weak measurements do not effectively become a strong measurement before the system can evolve coherently.

The second trend is that optimal gain is larger for the nearest-neighbor approach than the imbalance approach. This is most likely a result of the fact that the uncertainty is larger for the imbalance approach. Since the imbalance approach uses five measurements for each tunneling link while the nearest-neighbor approach uses two, the relative uncertainties in the tunneling strength differ by roughly a factor of 2.5 [90], which is consistent with the observed behavior.

The third trend is that for the parameters and target states considered, the imbalance approach performs much better than the nearest-neighbor approach. The nearest-neighbor

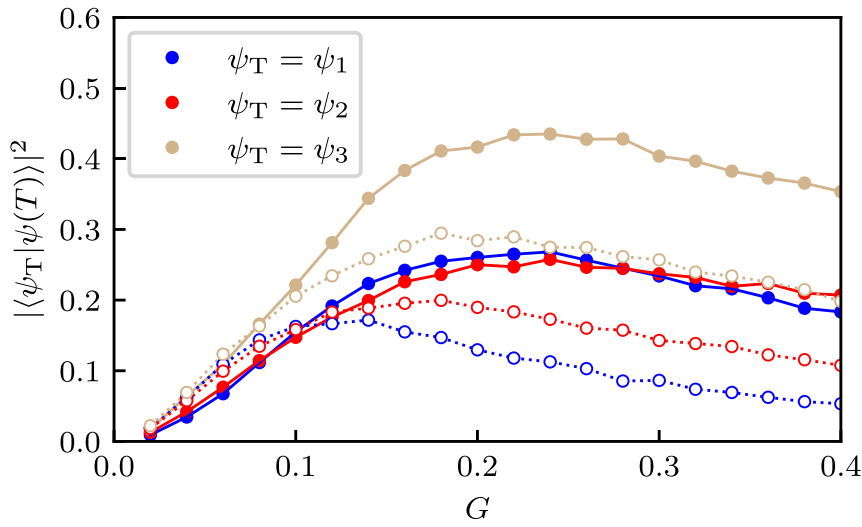

FIG. 6. Average overlap $\left|\left\langle\psi_{\mathrm{T}} \mid \psi(T)\right\rangle\right|^{2}$ for different values of $G$ when the initial states are close to the target state using 4096 trajectories. The solid (dotted) lines correspond to the nearest-neighbor (imbalance) approach. Blue, red, and tan lines correspond to $\psi_{T}=$ $\psi_{1}, \psi_{2}, \psi_{3}$, respectively.

approach performs better the more homogeneous the target state is. However, this begins to change at lower values of the measurement strength, where the target state overlap sharply begins to fall. This is a consequence of the fact that, at sufficiently small measurement strengths, the uncertainty in the measurement-and therefore the feedback signal—overwhelms the useful information. Since the imbalance approach involves more measurements, this behavior occurs earlier than for the nearest-neighbor approach.

\section{Nearby state preparation}

For larger systems, the effect of the increased uncertainty that comes with the imbalance approach becomes far more important. A natural approach to avoid this issue is to use a more hierarchical approach to the application of feedback. When the system starts initially far from the desired target state, a more global approach to feedback should be used. As the system gets closer to the target state, according to the measurement record, then increasingly local feedback approaches can be used.

While the systems we can consider numerically are too small to apply such a hierarchical feedback approach, we can explore how the performance of the two feedback approaches can change if the system is already closer to the target state. To do so, we consider initial states whose only difference from the target state is that a single boson has been moved one site away. Additionally, we allow the feedback to be applied for only one unit of time after the initialization of the measurement record (which requires time $\tau$ ), reflecting the fact that the local feedback would be applied for a shorter time in this hierarchical approach. The results of our numerics are shown in Fig. 6 for $\kappa=U$, demonstrating that the nearestneighbor approach performs better when the system is already close to the desired state due to the reduced uncertainty in the feedback. Note that this is in spite of the fact that the nearest-neighbor approach will initially turn on three coupling terms while the imbalance approach will turn on only one in the absence of measurement uncertainty. 


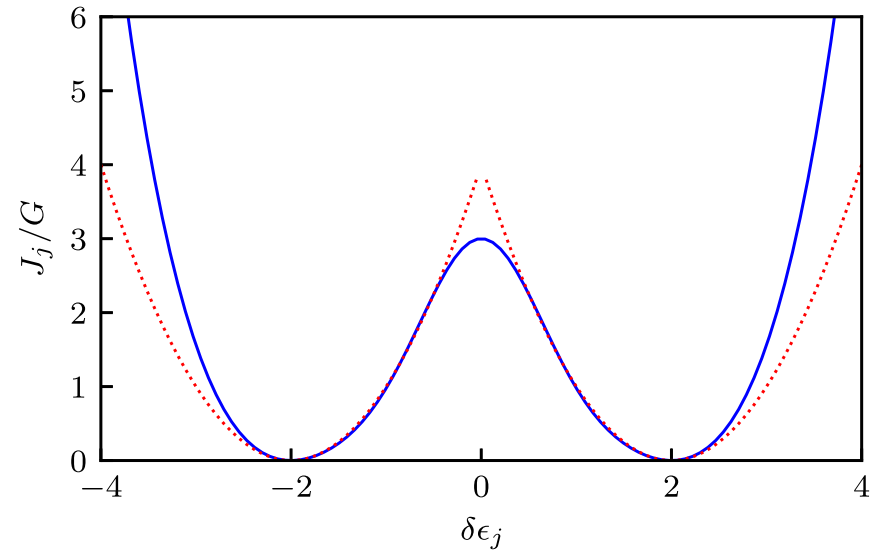

FIG. 7. Solid blue line shows tunneling feedback used for symmetry-breaking transition as a function of $\delta \epsilon_{j}$. Red dotted lines correspond to the feedback used in the state preparation section, with the left (right) curve corresponding to the target state |020202〉 $(|202020\rangle)$.

\section{Symmetry-breaking transition}

The emergence of phase transitions via feedback has been investigated in a variety of diverse contexts [41-49], and here we explore a symmetry-breaking transition in an extended Hubbard chain. To observe this, we will consider a six-site lattice with periodic boundaries whose tunneling feedback drives the system toward the states $|202020\rangle$ or $|020202\rangle$. Due to the periodic boundary conditions and type of symmetry breaking, the left-right imbalance approach is not applicable, so we consider a generalization of the nearest-neighbor feedback. In particular, the tunneling feedback is

$$
\begin{aligned}
J_{j}(t) & =\frac{G}{16}\left(1+2 e^{-\delta \epsilon_{j}^{2}}\right)\left(\delta \epsilon_{j}-2\right)^{2}\left(\delta \epsilon_{j}+2\right)^{2}, \\
\delta \epsilon_{j} & =\left[\epsilon_{j}(t)-\epsilon_{j+1}(t)\right],
\end{aligned}
$$

with $\epsilon_{7} \equiv \epsilon_{1}$. This is essentially a product of the tunneling feedback in Eq. (15) for $N_{j}-N_{j+1}= \pm 2$. The Gaussian term is included to increase the tunneling for $\epsilon_{j}-\epsilon_{j+1} \approx 0$ compared to just the quartic potential so it is comparable to the corresponding tunneling when only one of the two aforementioned states is a target state. This will serve as a barrier which hinders the system from moving from $|202020\rangle$ to $|020202\rangle$ and vice versa (see Fig. 7).

An additional important feature to note for this choice of feedback is that these are not the only two states which will lead to small tunneling. States of the form $|024200\rangle$ can also be relatively stable. While there is one pair of sites with large tunneling, this pair of sites has no bosons, so the large hopping is not sufficient on its own to change the state. However, there are two factors that can make such states unfavorable. The first is that the creation of these states requires four bosons on a single site, which is energetically unfavorable. The second is that once measurement-induced fluctuations cause one of the bosons at sites with two bosons to move into an empty site, the boson will quickly move to the next site.

To investigate the symmetry-breaking behavior of this feedback, we consider the behavior of the steady-state density matrix $\rho_{s s}$ for fixed $\kappa$ as a function of $G$, which we obtain

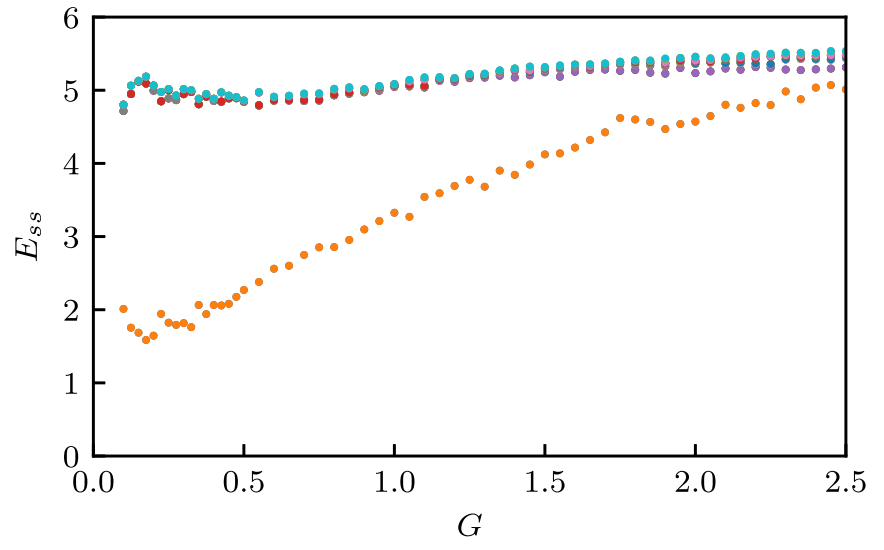

FIG. 8. Twenty smallest effective energies of steady-state $H_{s s}$ as a function of $G$. Eigenvalues are calculated after symmetrizing $\rho_{s s}$ with respect to translations and reflections, leading to degeneracy for states related through these symmetries; quantitatively similar behavior emerges without symmetrization, although the eigenvalues are no longer as degenerate. The orange dots are doubly degenerate and correspond approximately to $|202020\rangle,|020202\rangle$, with the strongest overlap occurring for the larger gaps. For $G<.5 U, \rho_{s s}$ is averaged over 128 trajectories from $t=100 U^{-1}$ to $t=400 U^{-1}$. For $G \geqslant .5 U, \rho_{s s}$ is averaged over 32 trajectories from $t=20 U^{-1}$ to $t=320 U^{-1}$. The initial time delay for averaging ensures that the system has relaxed to the steady state.

via a combination of ensemble and ergodic averaging. Additionally, we define an effective Hamiltonian $H_{s s}$ according to $e^{-H_{s s}} \equiv \rho_{s s}$. In analogy to equilibrium systems, a symmetrybreaking phase will occur when the two lowest energy levels of $H_{s s}$ become gapped from the rest of the spectrum. In terms of $\rho_{s s}$, the two corresponding states will have a much larger probability than the rest of the eigenstates, and the gap in $H_{S S}$ describes the exponential suppression in probability of these other eigenstates. Hence, in the absence of a gap, no particular configuration is favored, and the system appears disordered.

Figure 8 plots the eigenvalues of $H_{s s}$ as a function of $G$ for $\kappa=5 U$ using the initial state $\psi(0)=|111111\rangle$, which will prefer both symmetry-breaking states equally, although we expect the same steady state for Haar random initial states. We see that an effective gap opens for a range of $G$, with two effective energies much smaller than the rest. As expected, these two eigenvectors correspond approximately to $|202020\rangle$ and $|020202\rangle$, while the other possible stable states (e.g., $|024200\rangle)$ are not strongly occupied. Additionally, as this gap increases, the autocorrelation times increase, necessitating more sampling for larger gaps. This corresponds to the fact that when the system is in either of the two symmetry-broken states, the feedback makes it difficult to leave this state, so the system spends a long time in the same state, thus slowing down the ergodic sampling of $\rho_{s s}$. In the limit of large $G$, even small fluctuations will drastically modify $J_{j}(t)$, preventing the system from preferring any particular state and causing the gap to close.

As $G$ is increased, this gap eventually closes and does not reopen. Physically, this is because in the limit of large $G$, the hopping becomes very large, so small fluctuations in the measurements lead to large fluctuations in the hopping, 
preventing the system from preferring any given state. This is qualitatively similar to an infinite temperature limit in which no particular state is energetically preferred. In the limit of small $G$, the gap similarly closes because the measurement strength overwhelms the feedback, causing the system to relax to a random number state. The start of this closure can be observed in Fig. 8, although due to the quantum Zeno effect, identifying the steady state for smaller values of $G$ becomes numerically prohibitive as it requires increasingly long relaxation times.

\section{OUTLOOK}

This paper combines concepts from many-body physics with the techniques of quantum control and feedback to create and characterize low-entropy many-body dynamical steady states. Creating such a dynamical steady state in the laboratory environment hinges on exploring the implication of experimental realities, for example: What are the consequences of imperfect detection? Of limited spatial resolution? What is the impact of limited feedback bandwidth? Real implementation must face these questions head-on.

From a foundational perspective, it remains to be seen if there exist dynamical steady states which are forbidden in associated thermal equilibrium systems. For example, nonreciprocal couplings can be realized using feedback, which can lead to rich nonequilibrium phenomena [91-94]. Alternatively, feedback that is nonlocal in time can be used to engineer non-Markovian baths even though the measurements themselves are Markovian. Similarly, even with local measurements of density, nonlocal feedback can emulate aspects of long-range potentials [37]. Stochastic descriptions such as ours can be reframed in terms of the so-called feedback master equation [95], in which the time delay introduced by the low-pass filter introduces non-Markovian terms that cannot be described in a Lindblad form. This at least provides the opportunity for creating dynamics and steady states that are not achievable using realistic Lindblad terms. Moreover, even if the steady state may look thermal, its linear response may still violate the fluctuation-dissipation theorem [96,97], indicating the persistence of nonequilibrium features. Additionally, new types of dynamics are possible when the bosons include a spin degree of freedom, such as quantum Zeno-like effects which can emerge if one spin state is subjected to stronger measurements than the other.

While we have demonstrated how the use of nonlocal feedback can be employed to prepare desired target states more efficiently than local feedback, this was done for the relatively simple case of Mott-like number states. An interesting next direction, then, is to consider preparing more complex states, such as superfluidlike states with long-range coherences. By adding density modulations through the use of nonlocal feedback as we did for the number states, this opens the possibility of preparing supersolid and supersolid-like states [98-101]. More broadly, this would provide further insight into how the use of measurement and feedback affects the ability to realize intrinsically quantum dynamics.

Although we have illustrated how a symmetry-breaking phase transition may in principal emerge due to measurement and feedback in a many-body quantum system, several open questions remain. Here, our analysis has been restricted to small 1D chains, so an important question is whether this corresponds to a phase transition in the thermodynamic limit. Even if it does not exist in 1D, an analogous transition may exist in higher-dimensional systems. Additionally, understanding the behavior of critical phenomena in these systems is another important direction, such as whether they are equivalent to a quantum phase transition, a classical phase transition, or something entirely different. In Ref. [46], it was shown that in zero-dimensional systems, modifying the form of non-Markovian feedback can lead to changes in the critical exponents of the phase transition, so similar phenomena may arise in a many-body context as well.

A natural direction to explore to investigate these questions are measurement-induced phase transitions, which have been the subject of intense theoretical research in recent years and are defined by the scaling behavior of the entanglement entropy [102-118] with recent initial experimental evidence [119]. Aside from the key difference that these systems are not subject to feedback, these often involve coherent evolution defined by random unitary circuits and strong measurements, although similar behavior has been shown to emerge for weak measurements as well [114-118]. A natural question is how these phase transitions can be modified through different applications of feedback: Will the phase transition only shift or can qualitatively new physics emerge?

Another promising approach to exploring the above questions is to investigate the similarities that systems subject to measurement and feedback have with driven-dissipative systems, which are systems that are subjected to coherent drive and incoherent dissipation that have been studied extensively in a variety of contexts [1-12,93,120-136]. From an abstract standpoint, these two types of systems are very similar, with dissipation playing a role analogous to continuous measurements and drive playing a role analogous to the feedback. As a result, insights or techniques from studying one type of system can lead to insights in the other. For example, phase transitions in driven-dissipative systems have been studied extensively using a Keldysh-Schwinger and functional integral formalism [93,122-131], so it is important to explore how these same techniques can be applied to systems subject to measurement and feedback. Similarly, effective classical equilibrium criticality is observed generically in many driven-dissipative phase transitions [127-129], with some important exceptions [93,130-132], so measurementfeedback systems may provide new avenues to realize novel forms of nonequilibrium criticality and quantum criticality. Moreover, recent research indicates that dissipative phase transitions and measurement-induced phase transitions need not coincide [136], which means that measurement-feedback phase transitions may similarly lead to distinctive forms of criticality.

\section{ACKNOWLEDGMENTS}

We benefited greatly from conversations with H. M. Hurst, J. K. Thompson, J. Ye, L. Walker, R. Lena, A. Daley, L. P. García-Pintos, S. Guo, and E. Altuntas. We appreciate the careful reading from $\mathrm{S}$. Lieu and D. Barberena. J.T.Y. 
was supported by the NIST NRC Research Postdoctoral Associateship Award. A.V.G. acknowledges funding by DARPA SAVaNT ADVENT, AFOSR, ARO MURI, AFOSR MURI, U.S. Department of Energy Award No. DESC0019449, NSF PFCQC program, DoE ASCR Accelerated
Research in Quantum Computing program (Award No. DESC0020312), and DoE ASCR Quantum Testbed Pathfinder program (Award No. DE-SC0019040). This work was supported by NIST. Analysis was performed in part on the NIST Raritan HPC cluster.
[1] S. Diehl, A. Micheli, A. Kantian, B. Kraus, H. P. Büchler, and P. Zoller, Quantum states and phases in driven open quantum systems with cold atoms, Nat. Phys. 4, 878 (2008).

[2] M. H. Szymańska, J. Keeling, and P. B. Littlewood, Meanfield theory and fluctuation spectrum of a pumped decaying Bose-Fermi system across the quantum condensation transition, Phys. Rev. B 75, 195331 (2007).

[3] W. Yi, S. Diehl, A. J. Daley, and P. Zoller, Driven-dissipative many-body pairing states for cold fermionic atoms in an optical lattice, New J. Phys. 14, 055002 (2012).

[4] C.-E. Bardyn, M. A. Baranov, C. V. Kraus, E. Rico, A. İmamoğlu, P. Zoller, and S. Diehl, Topology by dissipation, New J. Phys. 15, 085001 (2013).

[5] K. Baumann, C. Guerlin, F. Brennecke, and T. Esslinger, Dicke quantum phase transition with a superfluid gas in an optical cavity, Nature (London) 464, 1301 (2010).

[6] C. Carr, R. Ritter, C. G. Wade, C. S. Adams, and K. J. Weatherill, Nonequilibrium Phase Transition in a Dilute Rydberg Ensemble, Phys. Rev. Lett. 111, 113901 (2013).

[7] N. Malossi, M. M. Valado, S. Scotto, P. Huillery, P. Pillet, D. Ciampini, E. Arimondo, and O. Morsch, Full Counting Statistics and Phase Diagram of a Dissipative Rydberg Gas, Phys. Rev. Lett. 113, 023006 (2014).

[8] J. Jin, A. Biella, O. Viyuela, L. Mazza, J. Keeling, R. Fazio, and D. Rossini, Cluster Mean-Field Approach to the SteadyState Phase Diagram of Dissipative Spin Systems, Phys. Rev. X 6, 031011 (2016).

[9] S. R. K. Rodriguez, W. Casteels, F. Storme, N. Carlon Zambon, I. Sagnes, L. Le Gratiet, E. Galopin, A. Lemaître, A. Amo, C. Ciuti, and J. Bloch, Probing a Dissipative Phase Transition Via Dynamical Optical Hysteresis, Phys. Rev. Lett. 118, 247402 (2017).

[10] M. Fitzpatrick, N. M. Sundaresan, A. C. Y. Li, J. Koch, and A. A. Houck, Observation of a Dissipative Phase Transition in a One-Dimensional Circuit QED Lattice, Phys. Rev. X 7, 011016 (2017).

[11] N. Dogra, M. Landini, K. Kroeger, L. Hruby, T. Donner, and T. Esslinger, Dissipation-induced structural instability and chiral dynamics in a quantum gas, Science 366, 1496 (2019).

[12] O. Scarlatella, A. A. Clerk, R. Fazio, and M. Schiró, Dynamical Mean-Field Theory for Markovian Open Quantum Many-Body Systems, Phys. Rev. X 11, 031018 (2021).

[13] A. C. Cassidy, C. W. Clark, and M. Rigol, Generalized Thermalization in an Integrable Lattice System, Phys. Rev. Lett. 106, 140405 (2011).

[14] T. Langen, S. Erne, R. Geiger, B. Rauer, T. Schweigler, M. Kuhnert, W. Rohringer, I. E. Mazets, T. Gasenzer, and J. Schmiedmayer, Experimental observation of a generalized Gibbs ensemble, Science 348, 207 (2015).

[15] E. Ilievski, M. Medenjak, T. Prosen, and L. Zadnik, Quasilocal charges in integrable lattice systems, J. Stat. Mech.: Theory Exp. (2016) 064008.
[16] P. Calabrese, F. H. L. Essler, and G. Mussardo, Introduction to 'quantum integrability in out of equilibrium systems', J. Stat. Mech.: Theory Exp. (2016) 064001.

[17] L. Vidmar and M. Rigol, Generalized Gibbs ensemble in integrable lattice models, J. Stat. Mech.: Theory Exp. (2016) 064007.

[18] T. Mori, T. N. Ikeda, E. Kaminishi, and M. Ueda, Thermalization and prethermalization in isolated quantum systems: A theoretical overview, J. Phys. B: At., Mol. Opt. Phys. 51, 112001 (2018).

[19] T. Kinoshita, T. Wenger, and D. S. Weiss, A quantum Newton's cradle, Nature (London) 440, 900 (2006).

[20] M. Gring, M. Kuhnert, T. Langen, T. Kitagawa, B. Rauer, M. Schreitl, I. Mazets, D. A. Smith, E. Demler, and J. Schmiedmayer, Relaxation and prethermalization in an isolated quantum system, Science 337, 1318 (2012).

[21] Y. Tang, W. Kao, K.-Y. Li, S. Seo, K. Mallayya, M. Rigol, S. Gopalakrishnan, and B. L. Lev, Thermalization Near integrability in a Dipolar Quantum Newton's Cradle, Phys. Rev. X 8, 021030 (2018).

[22] W. Wu, B. Ellman, T. F. Rosenbaum, G. Aeppli, and D. H. Reich, From Classical to Quantum Glass, Phys. Rev. Lett. 67, 2076 (1991).

[23] S. Gopalakrishnan, B. L. Lev, and P. M. Goldbart, Frustration and Glassiness in Spin Models with Cavity-Mediated Interactions, Phys. Rev. Lett. 107, 277201 (2011).

[24] E. Altman and R. Vosk, Universal dynamics and renormalization in many-body-localized systems, Annu. Rev. Condens. Matter Phys. 6, 383 (2015).

[25] R. Nandkishore and D. A. Huse, Many-body localization and thermalization in quantum statistical mechanics, Annu. Rev. Condens. Matter Phys. 6, 15 (2015).

[26] C. Gogolin and J. Eisert, Equilibration, thermalisation, and the emergence of statistical mechanics in closed quantum systems, Rep. Prog. Phys. 79, 056001 (2016).

[27] F. Alet and N. Laflorencie, Many-body localization: An introduction and selected topics, C. R. Phys. 19, 498 (2018).

[28] D. A. Abanin, E. Altman, I. Bloch, and M. Serbyn, Colloquium: Many-body localization, thermalization, and entanglement, Rev. Mod. Phys. 91, 021001 (2019).

[29] M. Schreiber, S. S. Hodgman, P. Bordia, H. P. Luschen, M. H. Fischer, R. Vosk, E. Altman, U. Schneider, and I. Bloch, Observation of many-body localization of interacting fermions in a quasirandom optical lattice, Science 349, 842 (2015).

[30] J.-y. Choi, S. Hild, J. Zeiher, P. Schauß, A. Rubio-Abadal, T. Yefsah, V. Khemani, D. A. Huse, I. Bloch, and C. Gross, Exploring the many-body localization transition in two dimensions, Science 352, 1547 (2016).

[31] J. Smith, A. Lee, P. Richerme, B. Neyenhuis, P. W. Hess, P. Hauke, M. Heyl, D. A. Huse, and C. Monroe, Many-body localization in a quantum simulator with programmable random disorder, Nat. Phys. 12, 907 (2016). 
[32] P. Roushan, C. Neill, J. Tangpanitanon, V. M. Bastidas, A. Megrant, R. Barends, Y. Chen, Z. Chen, B. Chiaro, A. Dunsworth, A. Fowler, B. Foxen, M. Giustina, E. Jeffrey, J. Kelly, E. Lucero, J. Mutus, M. Neeley, C. Quintana, D. Sank, A. Vainsencher, J. Wenner, T. White, H. Neven, D. G. Angelakis, and J. Martinis, Spectroscopic signatures of localization with interacting photons in superconducting qubits, Science 358, 1175 (2017)

[33] Q. Guo, C. Cheng, Z.-H. S. Z. Song, H. Li, Z. Wang, W. Ren, H. Dong, D. Zheng, Y.-R. Zhang, R. Mondaini, H. Fan, and H. Wang, Observation of energy-resolved many-body localization, Nat. Phys. 17, 234 (2021).

[34] S. Lloyd and Jean-Jacques E. Slotine, Quantum feedback with weak measurements, Phys. Rev. A 62, 012307 (2000).

[35] L. K. Thomsen, S. Mancini, and H. M. Wiseman, Spin squeezing via quantum feedback, Phys. Rev. A 65, 061801(R) (2002).

[36] A. Shankar, G. P. Greve, B. Wu, J. K. Thompson, and M. Holland, Continuous Real-Time Tracking of a Quantum Phase Below the Standard Quantum Limit, Phys. Rev. Lett. 122, 233602 (2019).

[37] C. A. Muschik, K. Hammerer, E. S. Polzik, and I. J. Cirac, Quantum Teleportation of Dynamics and Effective Interactions Between Remote Systems, Phys. Rev. Lett. 111, 020501 (2013).

[38] M. H. Muñoz-Arias, P. M. Poggi, P. S. Jessen, and I. H. Deutsch, Simulating Nonlinear Dynamics of Collective Spins Via Quantum Measurement and Feedback, Phys. Rev. Lett. 124, 110503 (2020).

[39] L.-N. Wu and A. Eckardt, Cooling and state preparation in an optical lattice via Markovian feedback control, arXiv:2106.03883.

[40] H. M. Hurst and I. B. Spielman, Measurement-induced dynamics and stabilization of spinor-condensate domain walls, Phys. Rev. A 99, 053612 (2019).

[41] H. M. Hurst, S. Guo, and I. B. Spielman, Feedback induced magnetic phases in binary Bose-Einstein condensates, Phys. Rev. Research 2, 043325 (2020).

[42] A. L. Grimsmo, A. S. Parkins, and B.-S. Skagerstam, Rapid steady-state convergence for quantum systems using timedelayed feedback control, New J. Phys. 16, 065004 (2014).

[43] W. Kopylov, C. Emary, E. Schöll, and T. Brandes, Timedelayed feedback control of the Dicke-Hepp-Lieb superradiant quantum phase transition, New J. Phys. 17, 013040 (2015).

[44] G. Mazzucchi, S. F. Caballero-Benitez, D. A. Ivanov, and I. B. Mekhov, Quantum optical feedback control for creating strong correlations in many-body systems, Optica 3, 1213 (2016).

[45] M. H. Muñoz-Arias, I. H. Deutsch, P. S. Jessen, and P. M. Poggi, Simulation of the complex dynamics of mean-field $p$-spin models using measurement-based quantum feedback control, Phys. Rev. A 102, 022610 (2020).

[46] D. A. Ivanov, T. Y. Ivanova, S. F. Caballero-Benitez, and I. B. Mekhov, Feedback-Induced Quantum Phase Transitions Using Weak Measurements, Phys. Rev. Lett. 124, 010603 (2020).

[47] K. Kroeger, N. Dogra, R. Rosa-Medina, M. Paluch, F. Ferri, T. Donner, and T. Esslinger, Continuous feedback on a quantum gas coupled to an optical cavity, New J. Phys. 22, 033020 (2020).
[48] D. A. Ivanov, T. Y. Ivanova, S. F. Caballero-Benitez, and I. B. Mekhov, Cavityless self-organization of ultracold atoms due to the feedback-induced phase transition, Sci. Rep. 10, 10550 (2020).

[49] D. A. Ivanov, T. Y. Ivanova, S. F. Caballero-Benitez, and I. B. Mekhov, Tuning the universality class of phase transitions by feedback: Open quantum systems beyond dissipation, Phys. Rev. A 104, 033719 (2021).

[50] W. Happer, Optical pumping, Rev. Mod. Phys. 44, 169 (1972).

[51] P. D. Lett, R. N. Watts, C. I. Westbrook, W. D. Phillips, P. L. Gould, and H. J. Metcalf, Observation of Atoms Laser Cooled Below the Doppler Limit, Phys. Rev. Lett. 61, 169 (1988).

[52] P. W. Shor, Scheme for reducing decoherence in quantum computer memory, Phys. Rev. A 52, R2493 (1995).

[53] B. M. Terhal, Quantum error correction for quantum memories, Rev. Mod. Phys. 87, 307 (2015).

[54] S. J. Devitt, W. J. Munro, and K. Nemoto, Quantum error correction for beginners, Rep. Prog. Phys. 76, 076001 (2013).

[55] V. Giovannetti, S. Lloyd, and L. Maccone, Advances in quantum metrology, Nat. Photonics 5, 222 (2011).

[56] C. L. Degen, F. Reinhard, and P. Cappellaro, Quantum sensing, Rev. Mod. Phys. 89, 035002 (2017).

[57] D. F. Walls, Squeezed states of light, Nature (London) 306, 141 (1983).

[58] M. Kitagawa and M. Ueda, Squeezed spin states, Phys. Rev. A 47, 5138 (1993).

[59] M. H. Schleier-Smith, I. D. Leroux, and V. Vuletić, States of an Ensemble of Two-Level Atoms with Reduced Quantum Uncertainty, Phys. Rev. Lett. 104, 073604 (2010).

[60] O. Hosten, N. J. Engelsen, R. Krishnakumar, and M. A. Kasevich, Measurement noise 100 times lower than the quantum-projection limit using entangled atoms, Nature (London) 529, 505 (2016).

[61] K. C. Cox, G. P. Greve, J. M. Weiner, and J. K. Thompson, Deterministic Squeezed States with Collective Measurements and Feedback, Phys. Rev. Lett. 116, 093602 (2016).

[62] A. N. Korotkov, Selective quantum evolution of a qubit state due to continuous measurement, Phys. Rev. B 63, 115403 (2001).

[63] C. Ahn, A. C. Doherty, and A. J. Landahl, Continuous quantum error correction via quantum feedback control, Phys. Rev. A 65, 042301 (2002).

[64] M. Sarovar, C. Ahn, K. Jacobs, and G. J. Milburn, Practical scheme for error control using feedback, Phys. Rev. A 69, 052324 (2004).

[65] W. Dür, M. Skotiniotis, F. Fröwis, and B. Kraus, Improved Quantum Metrology Using Quantum Error Correction, Phys. Rev. Lett. 112, 080801 (2014).

[66] E. M. Kessler, I. Lovchinsky, A. O. Sushkov, and M. D. Lukin, Quantum Error Correction for Metrology, Phys. Rev. Lett. 112, 150802 (2014).

[67] G. Arrad, Y. Vinkler, D. Aharonov, and A. Retzker, Increasing Sensing Resolution with Error Correction, Phys. Rev. Lett. 112, 150801 (2014).

[68] T. Unden, P. Balasubramanian, D. Louzon, Y. Vinkler, M. B. Plenio, M. Markham, D. Twitchen, A. Stacey, I. Lovchinsky, A. O. Sushkov, M. D. Lukin, A. Retzker, B. Naydenov, L. P. McGuinness, and F. Jelezko, Quantum Metrology Enhanced by Repetitive Quantum Error Correction, Phys. Rev. Lett. 116, 230502 (2016). 
[69] P. Sekatski, M. Skotiniotis, J. Kołodyński, and W. Dür, Quantum metrology with full and fast quantum control, Quantum 1, 27 (2017).

[70] F. Reiter, A. S. Sørensen, P. Zoller, and C. A. Muschik, Dissipative quantum error correction and application to quantum sensing with trapped ions, Nat. Commun. 8, 1822 (2017).

[71] S. Zhou, M. Zhang, J. Preskill, and L. Jiang, Achieving the Heisenberg limit in quantum metrology using quantum error correction, Nat. Commun. 9, 78 (2018).

[72] D. Layden, S. Zhou, P. Cappellaro, and L. Jiang, Ancilla-Free Quantum Error Correction Codes for Quantum Metrology, Phys. Rev. Lett. 122, 040502 (2019).

[73] K. Jacobs and D. A. Steck, A straightforward introduction to continuous quantum measurement, Contemp. Phys. 47, 279 (2006).

[74] A. A. Clerk, M. H. Devoret, S. M. Girvin, F. Marquardt, and R. J. Schoelkopf, Introduction to quantum noise, measurement, and amplification, Rev. Mod. Phys. 82, 1155 (2010).

[75] M. R. Hush, S. S. Szigeti, A. R. R. Carvalho, and J. J. Hope, Controlling spontaneous-emission noise in measurementbased feedback cooling of a Bose-Einstein condensate, New J. Phys. 15, 113060 (2013).

[76] M. A. Nielsen and I. L. Chuang, Quantum Computation and Quantum Information (Cambridge University Press, Cambridge, 2010).

[77] J. Van de Vegte, Feedback Control Systems, 3rd ed., Prentice Hall International Editions (Prentice Hall, New Jersey, USA, 1994).

[78] R. E. Kalman, A new approach to linear filtering and prediction problems, Trans. ASME-J. Basic Eng. 82, 35 (1960).

[79] W. S. Bakr, J. I. Gillen, A. Peng, S. Fölling, and M. Greiner, A quantum gas microscope for detecting single atoms in a Hubbard-regime optical lattice, Nature (London) 462, 74 (2009).

[80] J. F. Sherson, C. Weitenberg, M. Endres, M. Cheneau, I. Bloch, and S. Kuhr, Single-atom-resolved fluorescense imaging of an atomic Mott insulator, Nature (London) 467, 68 (2010).

[81] R. Islam, R. Ma, P. M. Preiss, M. Eric Tai, A. Lukin, M. Rispoli, and M. Greiner, Measuring entanglement entropy in a quantum many-body system, Nature (London) 528, 77 (2015).

[82] The factor of $\sqrt{2}$ derives from combining two measurements of density into a single measurement of $F_{z}$.

[83] S. Levy, E. Lahoud, I. Shomroni, and J. Steinhauer, The a.c. and d.c. Josephson effects in a Bose-Einstein condensate, Nature (London) 449, 579 (2007).

[84] R. Gati and M. K. Oberthaler, A bosonic Josephson junction, J. Phys. B 40, R61 (2007).

[85] M. Albiez, R. Gati, J. Fölling, S. Hunsmann, M. Cristiani, and M. K. Oberthaler, Direct Observation of Tunneling and Nonlinear Self-Trapping in a Single Bosonic Josephson Junction, Phys. Rev. Lett. 95, 010402 (2005).

[86] J. Dalibard, Y. Castin, and K. Mølmer, Wave-Function Approach to Dissipative Processes in Quantum Optics, Phys. Rev. Lett. 68, 580 (1992)

[87] M. B. Plenio and P. L. Knight, The quantum-jump approach to dissipative dynamics in quantum optics, Rev. Mod. Phys. 70, 101 (1998).

[88] R. Dum, P. Zoller, and H. Ritsch, Monte Carlo simulation of the atomic master equation for spontaneous emission, Phys. Rev. A 45, 4879 (1992).
[89] The extra time $\tau$ is to allow for an initialization of $\epsilon_{j}$ before the feedback starts being applied at $t=\tau$.

[90] For white noise $\xi$ with standard deviation $\sigma$, the mean and standard deviation of $\xi^{2}$ are $\sigma^{2}$ and $\sqrt{2} \sigma^{2}$, respectively, hence the factor of 2.5 rather than $\sqrt{2.5}$.

[91] A. Metelmann and A. A. Clerk, Nonreciprocal Photon Transmission and Amplification Via Reservoir Engineering, Phys. Rev. X 5, 021025 (2015).

[92] A. Metelmann and A. A. Clerk, Nonreciprocal quantum interactions and devices via autonomous feedforward, Phys. Rev. A 95, 013837 (2017).

[93] J. T. Young, A. V. Gorshkov, M. Foss-Feig, and M. F. Maghrebi, Non-Equilibrium Fixed Points of Coupled Ising Models, Phys. Rev. X 10, 011039 (2020).

[94] M. Fruchart, R. Hanai, P. B. Littlewood, and V. Vitelli, Nonreciprocal phase transitions, Nature (London) 592, 363 (2021).

[95] H. M. Wiseman and G. J. Milburn, Quantum Measurement and Control (Cambridge University Press, Cambridge, UK, 2009).

[96] H. B. Callen and T. A. Welton, Irreversibility and generalized noise, Phys. Rev. 83, 34 (1951).

[97] R. Kubo, The fluctuation-dissipation theorem, Rep. Prog. Phys. 29, 306 (1966).

[98] G. V. Chester, Speculations on Bose-Einstein condensation and quantum crystals, Phys. Rev. A 2, 256 (1970).

[99] M. Boninsegni and N. V. Prokof'ev, Colloquium: Supersolids: What and where are they? Rev. Mod. Phys. 84, 759 (2012).

[100] J. Léonard, A. Morales, P. Zupancic, T. Esslinger, and T. Donner, Supersolid formation in a quantum gas breaking a continuous translational symmetry, Nature (London) $\mathbf{5 4 3}, 87$ (2017).

[101] J.-r. Li, J. Lee, W. Huang, S. Burchesky, B. Shteynas, F. Ç. Top, A. O. Jamison, and W. Ketterle, A stripe phase with supersolid properties in spin-orbit-coupled Bose-Einstein condensates, Nature (London) 543, 91 (2017).

[102] Y. Li, X. Chen, and M. P. A. Fisher, Quantum Zeno effect and the many-body entanglement transition, Phys. Rev. B 98, 205136 (2018).

[103] Y. Li, X. Chen, and M. P. A. Fisher, Measurement-driven entanglement transition in hybrid quantum circuits, Phys. Rev. B 100, 134306 (2019)

[104] B. Skinner, J. Ruhman, and A. Nahum, Measurement-Induced Phase Transitions in the Dynamics of Entanglement, Phys. Rev. X 9, 031009 (2019).

[105] A. Chan, R. M. Nandkishore, M. Pretko, and G. Smith, Unitary-projective entanglement dynamics, Phys. Rev. B 99, 224307 (2019).

[106] S. Choi, Y. Bao, X.-L. Qi, and E. Altman, Quantum Error Correction in Scrambling Dynamics and Measurement-Induced Phase Transition, Phys. Rev. Lett. 125, 030505 (2020).

[107] C.-M. Jian, Y.-Z. You, R. Vasseur, and A. W. W. Ludwig, Measurement-induced criticality in random quantum circuits, Phys. Rev. B 101, 104302 (2020).

[108] X. Turkeshi, R. Fazio, and M. Dalmonte, Measurementinduced criticality in $(2+1)$-dimensional hybrid quantum circuits, Phys. Rev. B 102, 014315 (2020).

[109] M. J. Gullans and D. A. Huse, Dynamical Purification Phase Transition Induced by Quantum Measurements, Phys. Rev. X 10, 041020 (2020).

[110] M. Ippoliti, M. J. Gullans, S. Gopalakrishnan, D. A. Huse, and V. Khemani, Entanglement Phase Transitions in 
Measurement-Only Dynamics, Phys. Rev. X 11, 011030 (2021).

[111] A. Lavasani, Y. Alavirad, and M. Barkeshli, Measurementinduced topological entanglement transitions in symmetric random quantum circuits, Nat. Phys. 17, 342 (2021).

[112] A. Nahum, S. Roy, B. Skinner, and J. Ruhman, Measurement and entanglement phase transitions in all-to-all quantum circuits, on quantum trees, and in Landau-Ginsburg theory, PRX Quantum 2, 010352 (2021).

[113] T. Minato, K. Sugimoto, T. Kuwahara, and K. Saito, Fate of measurement-induced phase transition in long-range interactions, arXiv:2104.09118.

[114] X. Cao, A. Tilloy, and A. D. Luca, Entanglement in a fermion chain under continuous monitoring, SciPost Phys. 7, 24 (2019).

[115] M. Szyniszewski, A. Romito, and H. Schomerus, Entanglement transition from variable-strength weak measurements, Phys. Rev. B 100, 064204 (2019).

[116] Y. Bao, S. Choi, and E. Altman, Theory of the phase transition in random unitary circuits with measurements, Phys. Rev. B 101, 104301 (2020).

[117] S. Goto and I. Danshita, Measurement-induced transitions of the entanglement scaling law in ultracold gases with controllable dissipation, Phys. Rev. A 102, 033316 (2020).

[118] O. Alberton, M. Buchhold, and S. Diehl, Entanglement Transition in a Monitored Free-Fermion Chain: From Extended Criticality to Area Law, Phys. Rev. Lett. 126, 170602 (2021).

[119] C. Noel, P. Niroula, D. Zhu, A. Risinger, L. Egan, D. Biswas, M. Cetina, A. V. Gorshkov, M. J. Gullans, D. A. Huse, and C. Monroe, Observation of measurement-induced quantum phases in a trapped-ion quantum computer, arXiv:2106.05881.

[120] F. Minganti, A. Biella, N. Bartolo, and C. Ciuti, Spectral theory of Liouvillians for dissipative phase transitions, Phys. Rev. A 98, 042118 (2018).

[121] M. Soriente, T. Donner, R. Chitra, and O. Zilberberg, Dissipation-Induced Anomalous Multicritical Phenomena, Phys. Rev. Lett. 120, 183603 (2018).

[122] E. G. Dalla Torre, E. Demler, T. Giamarchi, and E. Altman, Quantum critical states and phase transitions in the presence of non-equilibrium noise, Nat. Phys. 6, 806 (2010).

[123] S. Gopalakrishnan, B. L. Lev, and P. M. Goldbart, Atom-light crystallization of Bose-Einstein condensates in multimode cavities: Nonequilibrium classical and quantum phase transi- tions, emergent lattices, supersolidity, and frustration, Phys. Rev. A 82, 043612 (2010).

[124] U. C. Täuber and S. Diehl, Perturbative Field-Theoretical Renormalization Group Approach to Driven-Dissipative BoseEinstein Criticality, Phys. Rev. X 4, 021010 (2014).

[125] E. Altman, L. M. Sieberer, L. Chen, S. Diehl, and J. Toner, Two-Dimensional Superfluidity of Exciton Polaritons Requires Strong Anisotropy, Phys. Rev. X 5, 011017 (2015).

[126] L. M. Sieberer, M. Buchhold, and S. Diehl, Keldysh field theory for driven open quantum systems, Rep. Prog. Phys. 79, 096001 (2016).

[127] Emanuele G. Dalla Torre, S. Diehl, M. D. Lukin, S. Sachdev, and P. Strack, Keldysh approach for nonequilibrium phase transitions in quantum optics: Beyond the Dicke model in optical cavities, Phys. Rev. A 87, 023831 (2013).

[128] M. F. Maghrebi and A. V. Gorshkov, Nonequilibrium manybody steady states via Keldysh formalism, Phys. Rev. B 93, 014307 (2016).

[129] M. Foss-Feig, P. Niroula, J. T. Young, M. Hafezi, A. V. Gorshkov, R. M. Wilson, and M. F. Maghrebi, Emergent equilibrium in many-body optical bistability, Phys. Rev. A 95, 043826 (2017).

[130] J. Marino and S. Diehl, Driven Markovian Quantum Criticality, Phys. Rev. Lett. 116, 070407 (2016).

[131] D. A. Paz and M. F. Maghrebi, Driven-dissipative Ising Model: An exact field-theoretical analysis, Phys. Rev. A 104, 023713 (2021).

[132] R. Rota, F. Minganti, C. Ciuti, and V. Savona, Quantum Critical Regime in a Quadratically Driven Nonlinear Photonic Lattice, Phys. Rev. Lett. 122, 110405 (2019).

[133] F. Iemini, A. Russomanno, J. Keeling, M. Schirò, M. Dalmonte, and R. Fazio, Boundary Time Crystals, Phys. Rev. Lett. 121, 035301 (2018).

[134] A. Nagy and V. Savona, Variational Quantum Monte Carlo Method with a Neural-Network Ansatz for Open Quantum Systems, Phys. Rev. Lett. 122, 250501 (2019).

[135] D. Roberts and A. A. Clerk, Driven-Dissipative Quantum Kerr Resonators: New Exact Solutions, Photon Blockade and Quantum Bistability, Phys. Rev. X 10, 021022 (2020).

[136] P. Sierant, G. Chiriacò, F. M. Surace, S. Sharma, X. Turkeshi, M. Dalmonte, R. Fazio, and G. Pagano, Dissipative Floquet dynamics: From steady state to measurement induced criticality in trapped-ion chains, arXiv:2107.05669. 